\title{
Lasers and optics: looking towards third generation gravitational wave detectors
}

\author{
Nergis Mavalvala • David E. McClelland • \\ Guido Mueller • D. H. Reitze • Roman Schnabel • \\ Benno Willke
}

Received: 18 August 2009 / Accepted: 19 May 2010 / Published online: 13 June 2010 (C) The Author(s) 2010. This article is published with open access at Springerlink.com

\begin{abstract}
Third generation terrestrial interferometric gravitational wave detectors will likely require significant advances in laser and optical technologies to reduce two of the main limiting noise sources: thermal noise due to mirror coatings and quantum noise arising from a combination of shot noise and radiation pressure noise. Increases in laser power and possible changes of the operational wavelength require new high power laser sources and new electro-optic modulators and Faraday isolators. Squeezed light can be used to further reduce the quantum noise while nano-structured optical components can be used to reduce or eliminate mirror coating thermal noise as well as to implement all-reflective interferometer configurations to avoid thermal effects in mirror substrates. This paper is intended to give an overview on the current state-of-the-art and future trends in these areas of ongoing research and development.
\end{abstract}

Keywords Gravitational wave detection · Laser interferometry

\footnotetext{
N. Mavalvala

LIGO Laboratory, Massachusetts Institute of Technology, Cambridge, MA 02139, USA

D. E. McClelland

ANU Centre for Gravitational Physics, Australian National University,

Canberra, ACT 0200, Australia

G. Mueller $(\varangle) \cdot$ D. H. Reitze

Department of Physics, University of Florida, Gainesville, FL 32611, USA

e-mail: mueller@phys.ufl.edu

R. Schnabel · B. Willke

Max-Planck-Institut für Gravitationsphysik (Albert-Einstein-Institut),

Leibniz Universität Hannover, 30167 Hannover, Germany
} 


\section{Introduction}

The next generation of ground-based interferometric gravitational wave detectors such as Advanced VIRGO and Advanced LIGO will be limited by thermal noise, quantum noise, and environmental low frequency noise such as seismic and Newtonian noise. Their ultimate sensitivity will be a compromise between low and high frequency performance [1]. Low power operation $(\sim 20 \mathrm{~W})$ reduces radiation pressure noise and the optical spring effect, resulting in improved low frequency sensitivity between 10 and $30 \mathrm{~Hz}$ while sacrificing high frequency sensitivity above $\sim 200 \mathrm{~Hz}$. Higher input power $(\sim 120 \mathrm{~W})$ has the opposite effect—it will improve the shot noise limit at high frequencies at the expense of increased radiation pressure noise at low frequencies.

From these fundamental considerations, it is likely that 3rd generation detectors have to compromise as well and that it will be necessary to build multiple detectors with overlapping frequency regions [2] to effectively cover the entire audio band. The 'xylophone' concept of co-located detectors could be realized with simple suspension systems in the current vacuum envelope of the LIGO and VIRGO detectors. In the high frequency range, more laser power and squeezing [3] are the tools of choice to reduce the shot noise limit. In addition, narrow-band signal recycling can further improve the sensitivity at specific frequencies at the expense of other frequencies outside of the bandwidth of the signal recycling cavity [4]. High frequency interferometers would improve the sensitivity at high frequencies where thermal noise falls below shot noise, however this requires more laser power and better power handling capabilities in the entire interferometer optical chain. Limitations in potential power build up inside the interferometer can then be overcome by more signal recycling gain (at the expense of detector bandwidth) and the need for additional narrow-band signal-recycled detectors and by squeezing. The implementation of squeezing requires the development of low loss Faraday isolators.

At lower frequencies, the answer is not as simple. Larger test masses will reduce radiation pressure noise but even the second generation detectors are only limited by radiation pressure noise in a small bandwidth $(\sim 15-30 \mathrm{~Hz})$. Below $\sim 10 \mathrm{~Hz}$ environmental noise sources become the limiting factor; the most fundamental of them, Newtonian (or gravity gradient) noise, is independent of the masses of the mirrors. Any increase in the masses requires a decrease in thermal noise to take advantage of the heavier mass [5], as well as improvements in suspension and seismic isolation systems, and finally reductions of Newtonian noise [6] by going to seismically quiet underground sites as well as adaptive sensing and cancellation of Newtonian noise. While improvements in suspension and seismic isolation systems and techniques to reduce or subtract Newtonian noise are unlikely to impact laser and optics development, substantial reductions in coating thermal noise may require changes in the laser wavelength.

Coating thermal noise is proportional to the thickness of the coatings and inversely proportional to the beam area. Standard coating layers have a thickness of $\lambda / 4 n$, where $\lambda$ is the free space wavelength of the laser. As the index of refraction $n$ of most coating materials is relatively constant in the near infrared (NIR) and visible spectral regions, coating thermal noise for a given beamsize is to first order proportional to $\lambda$. However, for the same cavity $g$-parameters, the beam area also scales as and the thermal noise is essentially independent of the wavelength. Shorter wavelength would allow to reduce 
the mirror size without increasing the thermal noise. This has potential applications in a xylophone type arrangement where multiple co-located detectors could cover the high frequency GW-band. One additional problem for the use of shorter wavelengths is the increased potential for photochemistry at the coating surfaces. The primary reason to consider a longer operational wavelength is in conjunction with the use of silicon as a test mass material. Silicon has very low absorption $(<1 \mathrm{ppm} / \mathrm{cm}$ at $\lambda>1.35 \mu \mathrm{m})$, has very low mechanical losses, is available in large sizes (potentially up to 100/kg masses) and is in principle the ideal test mass material. Silicon can also be used as a coating material. Its large index of refraction $(n=3.48)$ would reduce the thickness of each silicon layer and, paired up with a low index coating material such as $\mathrm{Si}_{2}(n=1.44)$, the required total number of layers would also be less than current optical coatings. Consequently, it is possible that the laser wavelength could either increase or decrease in third generation detectors.

This paper presents an evaluation of the current state-of-the-art in laser sources and optical components and discusses their extension to third generation ground-based detectors. The paper is organized as follows. In the next section, we give an overview of high power lasers and examine the possibilities for development of high power, stable sources at different wavelengths. Section 3 focuses on electro-optic modulators and Faraday isolators, with an emphasis on examining the available electro-optic and magneto-optic materials for short and long wavelength operation. Section 4 discusses novel trends in nanostructured optics for high power and low thermal noise operation. Section 5 presents squeezed light as a possible route to improved high frequency performance and summarizes the current status of squeezing as well as improvements needed for 3rd generation detectors.

\section{Light sources}

The current and the next generation of gravitational wave detectors use Nd:YAG lasers at $\lambda=1.064 \mu \mathrm{m}$ with output powers of up to $200 \mathrm{~W}$. The Advanced LIGO laser [7] consists of a four stage $\mathrm{Nd}$ :YVO amplifier system used to increase the $2 \mathrm{~W}$ power of a Nd:YAG non-planar ring-oscillator (NPRO) to $35 \mathrm{~W}$, followed by an injectionlocked Nd:YAG end-pumped rod ring cavity for final high power amplification. An output power of more than $210 \mathrm{~W}$ has been demonstrated in a linear-polarized, nearly diffraction limited, single-frequency operation [8]. The frequency stabilization of the laser will be accomplished by nested control loops beginning with a rigid-spacer reference cavity, followed by a longer mode-cleaner and the recycled interferometer as frequency references. Spatial filtering of the $200 \mathrm{~W}$ beam with a rigid spacer ring cavity will be used to bring the higher order mode content of the laser beam down to an acceptable level. The most demanding part of the laser stabilization is the relative power noise requirement of RPN $\leq 2 \times 10^{-9} / \sqrt{\mathrm{Hz}}$ at a frequency of $10 \mathrm{~Hz}$. This stability level has been recently achieved in a demonstration experiment using an NPRO with a multi-photodiode sensor and a sophisticated optical and electrical design [9].

The laser sources required for third generation detectors depend strongly on the optical configuration chosen. All-reflective interferometers have much higher power handling capabilities than current designs with transmissive optics. Sagnac 
interferometers [10] could operate with a laser source having low temporal coherence whereas configurations with optical cavities require a highly frequency stabilized laser. As noted above, coating thermal noise considerations might require a shorter wavelength whereas interferometers with transmissive silicon optics require lasers with longer wavelength. In addition, the preferred spatial beam profile might not be a fundamental Gaussian distribution but rather a flat-top profile or a higher order Laguerre Gaussian mode [11]. Even though thermal loading of the interferometer might ultimately limit the maximum power level in the interferometer, an increase in the laser power might eliminate the need for a power recycling mirror or reduce its reflectivity. In addition to this wide range of possibilities, several types of laser topologies as masterlaser power-amplifiers (MOPA), injection-locked systems or a mixture of both can be used to accommodate different types of gain-medium geometries such as slabs, rods, fibers or discs. Hence no definitive statement on the laser source for third generation detectors can be made and here we will only highlight some design considerations.

At the present time the Nd-doped YAG (Nd:YAG) gain medium is the best choice for $100 \mathrm{~W}$ class lasers. However, if kilowatt-class lasers are required for future detectors Yb-doped YAG, which lases at $1,030 \mathrm{~nm}$, may replace Nd:YAG because of its higher efficiency, lower quantum defect, better thermal management prospects and potentially longer-lived laser diode pumps. Its main disadvantages are i) that it is a quasi-3-level system and thus more sensitive to increased temperatures within the gain medium, and ii) that it has a much lower pump absorption coefficient.

Different design concepts have been proposed to produce lasers with power levels of several hundreds of Watts and to amplify these systems into the kilowatt region. The main concerns are the thermal management in the gain material to avoid stress-fracture damage and to reduce beam aberrations. A proven method for reducing aberrations uses a zig-zag beam path to average over the thermal gradient in the laser crystal [12]. Edge-pumped slab geometries can be combined with conduction-cooling techniques which avoid vibrations introduced by cooling fluids in conventional layouts $[13,14]$. One of the main challenges in using slabs is to avoid high gain parasitic beam paths.

Efficient birefringence compensation methods can reduce problems caused by depolarization and by defocusing in the gain medium due to thermal gradients. The power range in which rod geometries can be compensated can extend to several hundred watts. Besides 'treating' the effects caused by the thermal gradients there are several ideas to reduce these gradients. By using multi-segmented laser rods, the maximum peak temperature of an end-pumped laser rod or slab can be reduced. To decrease the overall heat load in Nd:YAG laser media, the pump wavelength can be changed from 807 to $885 \mathrm{~nm}$ which reduces the quantum defect and therefore the overall heat load by more than $30 \%$. Core-doped rods can be used to achieve an easier and more stable fundamental mode operation. These rods are comparable to a double clad fiber [15] where only the inner core of the rod is doped and the outer core is used as a waveguide for the pump light. As the gain is present only in the doped inner core of the rod, this concept is similar to mode selective pumping with the added advantage that a high brightness pump source is not required.

Optical fiber amplifiers have a great potential to offer single-frequency output at higher efficiencies and at lower cost than solid-state amplifiers at similar power levels (see [16] for an overview of high power fiber lasers). Until several years ago, 
diode-pumped fiber amplifiers were limited to power levels of several Watts due to the lack of high power single-mode pumps and to nonlinear effects in the fiber such as stimulated Raman scattering (SRS) and stimulated Brillouin scattering (SBS). The introduction of large mode-area double-clad (LMA) fibers and of photonic crystal fibers (PCF) has enabled output powers of single-mode fiber lasers to exceed $1 \mathrm{~kW}$ while retaining excellent conversion efficiencies [17]. The large effective core of these fibers decreases the average intensity in the fiber and thereby increases the threshold of nonlinear processes. The large inner cladding of the double clad fibers allows high power multi-mode pumps to be coupled into the fiber. Bending losses can be used to ensure that the output remains single-mode despite the large size of the core. The limiting factor for narrow-linewidth high-power fiber lasers for future use in gravitational wave detectors is the onset of SBS. A state-of-the art system with $150 \mathrm{~W}$ of output power with a good output beam profile (92\% in TEM00) is described in [18]. The optical-to-optical efficiency of this system with respect to incident pump power is $78 \%$ for a $195 \mathrm{~W}$ pump source. A good polarization ratio of approximately 100 to 1 has been achieved. Novel ideas to increase the SBS threshold are currently under investigation. One promising concept is to shift the Brillouin frequency along the fiber to lower the effective Brillouin gain for each frequency component. This can be achieved by temperature or strain gradients or varying doping concentrations along the fiber. In addition to the need to reduce nonlinear scattering, the reliability and noise performance of high power fiber lasers need to be further analyzed and possibly improved to meet the requirements of third generation detectors. One major advantage of fiber lasers is their compact physical footprint and simple, single stage architecture when compared with complex solid-state laser systems. Modern fiber splicing techniques can produce an all-fiber system including the master oscillator, the high power stage and possibly even a mode-cleaning fiber segment if required.

Considering longer wavelength, Erbium-doped fiber lasers emit around $1.53 \mu \mathrm{m}$ where the absorption in silicon is expected to be less than $0.1 \mathrm{ppm} / \mathrm{cm}$ [19]. The current state-of-the-art Er fiber systems include a master laser and a fiber amplifier and achieve output powers of $20 \mathrm{~W}$ [20] and much higher power levels are expected in the near future.

With respect to shorter wavelengths, lasers which emit directly in the visible include gas lasers (e.g. Argon ion) and dye lasers but their efficiency, reliability, controllability and noise performance rule them out as suitable lasers for gravitational wave detectors. Frequency-doubled Nd:YVO lasers operating at $532 \mathrm{~nm}$ are commercially available to powers approaching $20 \mathrm{~W}$, but have not been actively stabilized to required levels for gravitational wave interferometers. In case the interferometer design requires tunability or several closely spaced wavelengths Ti-Sapphire lasers could be chosen either at their fundamental wavelength $(650-1,070 \mathrm{~nm})$ or in a frequency-doubled layout. Frequency doubling or even tripling of high-power near-infrared lasers are a more promising option to provide high power sources at shorter wavelength. An attractive approach is the external second-harmonic-generation (SHG) in quasi-phase-matched ferroelectric materials such as $\mathrm{MgO}$-doped periodically poled $\mathrm{LiNbO}_{3}(\mathrm{MgO}: \mathrm{PPLN})$, $\mathrm{MgO}$-doped periodically poled stoichiometric $\mathrm{LiTaO}_{3}$ (MgO:sPPLT) and periodically poled $\mathrm{KTiOPO}_{4}$ (PPKTP). Green power levels of $16 \mathrm{~W}$ have been demonstrated by 
the conversion of a solid-state laser [21] and almost $10 \mathrm{~W}$ were achieved in a SHG experiment using an infrared fiber laser [22].

As many different applications drive the laser development worldwide, many laser concepts at different wavelength and power levels are available. Depending on the requirements of third generation gravitational wave detectors one of these designs can be chosen as the baseline for the light source. However, there is currently no application which has similar stringent requirements on the temporal and spatial stability as gravitational wave detectors. Hence a specific laser development program for third generation detectors will be required to design and build a reliable laser with sufficiently low free-running noise, an appropriate spatial beam profile and good controllability.

\section{Electro- and magneto-optical components}

The development of high power lasers at different wavelengths must be accompanied by the development of electro- and magneto-optical components capable of operating reliably at the increased powers. In current and next-generation gravitational wave detectors, electro-optical modulators are used to modulate the phase of the laser field with frequencies in the $5-100 \mathrm{MHz}$ range. The generated RF-sidebands are used to sense the various longitudinal and angular degrees of freedom of the interferometer. In current interferometers, the modulators are placed in air before the in-vacuum suspended mode cleaner(s). Therefore any distortions in the spatial profile or in the polarization will be filtered by the mode cleaner. This relaxes some of the requirements on the modulators significantly.

Faraday isolators are four-port optical components which use the Faraday effect to allow for non-reciprocal polarization switching of laser beams. They are used in current gravitational wave detectors in between amplifier stages of laser systems to suppress the backward propagation of light from high power stages into low power stages. These isolators transmit the power from the low power stages and are not as critical as Faraday isolators which operate in-vacuum. The in-vacuum isolators limit the amount of backward propagating light returning from the interferometer to prevent coupling into the source laser and back into the interferometer through secondary scattering. In addition, the returning light from the interferometer is routed to photodiodes and position sensors to provide diagnostic signals for length and alignment sensing and control. Faraday isolators have also been proposed for injecting squeezed light into the output port of interferometers to achieve sensing noise reduction [23].

\subsection{Electro-optical modulators}

In gravitational wave detectors, electro-optic modulators (EOMs) must be able to withstand the high laser powers without degrading the beam quality significantly. The main problems encountered in high power applications are photorefractive damage and variations in optical path length across the beam profile caused by the residual absorption of the laser beam. Photorefractive damage has a fairly well defined threshold in specific nonlinear crystals and can be increased by doping the crystals. Variations of 
Table 1 Material parameters at 1,064 nm for four widely used electro-optical materials

\begin{tabular}{lllll}
\hline & $\begin{array}{l}\mathrm{LiNbO}_{3} \\
\text { Uniaxial }\end{array}$ & $\begin{array}{l}\text { KTP } \\
\text { Biaxial }\end{array}$ & $\begin{array}{l}\text { RTP } \\
\text { Biaxial }\end{array}$ & $\begin{array}{l}\text { BBO } \\
\text { Uniaxial }\end{array}$ \\
\hline$r_{i j}(\mathrm{max})(\mathrm{pm} / \mathrm{V})$ & 32 & 37 & 39 & 2.7 \\
$n_{j}$ & 2.16 & 1.84 & 1.85 & 1.66 \\
$\beta=d n_{j} / d T(\mathrm{ppm} / \mathrm{K})$ & 39 & 15 & 11 & -9.3 \\
$\alpha_{j j}(\mathrm{ppm} / \mathrm{K})$ & 15 & 9 & 13 & 4 \\
$\kappa_{a a}(\mathrm{~min})(\mathrm{W} / \mathrm{mK})$ & 5.6 & 2 & 3 & 1.2 \\
$\gamma(1 / \mathrm{cm})$ & $1.5 \times 10^{-3}$ & $1.2 \times 10^{-4}$ & $10^{-4}$ & $10^{-3}$ \\
$\mathrm{FOM}(\mathrm{Wm} / \mathrm{V})$ & $2.7 \times 10^{-3}$ & $2.1 \times 10^{-2}$ & $4.1 \times 10^{-2}$ & $2.7 \times 10^{-4}$ \\
\hline
\end{tabular}

The thermal properties of $\mathrm{MgO}: \mathrm{LiNbO}_{3}$ are similar to the properties of $\mathrm{LiNbO}_{3}$ with the exception of the photorefractive damage threshold. BBO is a potential candidate for shorter wavelength. These parameters were extracted from several different references (see text and references) and should be used with caution as several of these values depend also on the specific composition of the crystal. For example $r_{33}$ of stoichiometric (mole ratio $\mathrm{Li} / \mathrm{Nb}=1) \mathrm{LiNbO}_{3}$ is $30 \%$ higher than of congruent (mole ratio $\left.\mathrm{Li} / \mathrm{Nb}=0.946\right) \mathrm{LiNbO}_{3}$ $[26]$

the optical path length (or equivalently short thermal lensing) are proportional to the length of the crystal and can be used to define a preliminary figure of merit (FOM) for various crystals:

$$
\mathrm{FOM}=\frac{4 \pi \kappa}{\alpha\left(n_{i}-1\right)+\beta} \frac{r_{i j} n_{i}^{3}}{\gamma}
$$

For a given applied RF-electric field polarized in the $j$-direction and a laser field polarized in the $i$-direction, the modulation index is proportional to the electro-optical coefficient $r_{i j}$ and the index of refraction $n_{i}$. The thermal lensing increases with the thermal expansion coefficient $\alpha$, the thermo-optical coefficient $\beta=\partial n / \partial T$, and the absorption coefficient $\gamma$. A large thermal conductivity $\kappa$ helps to diffuse the absorbed heat and reduces the thermal gradient [24].

This FOM does not incorporate damage threshold considerations (such as photorefractive damage) which limit the maximum allowed intensity in the crystals. An additional complication in some crystals arises from different thermal conductivities in the directions perpendicular to the propagation direction, creating a non-symmetric or cylindrical gradient index lens which is more difficult to compensate. Many of the material parameters even for widely used crystals vary in the open literature. Table 1 lists typical parameters for four types of crystals and also the derived FOM based on these parameters. A discussion of nonlinear crystals for use in EOMs and their potential application in high laser power interferometric gravitational wave detectors as well as references to the material parameters follows.

\section{$\mathrm{LiNbO}_{3}$}

Lithium niobate $\left(\mathrm{LiNbO}_{3}\right)$ is the most commonly used electro-optic material used in phase modulators in the near infrared and is used in the first generation of gravitational wave interferometers LIGO, VIRGO, GEO600, and TAMA. It is characterized by 
a fairly large electro-optical coefficient $r_{33}$ and index of refraction. Estimates for the thermal material parameters $\alpha$ and $\kappa$ of $\mathrm{LiNbO}_{3}$ are listed in Table 1 . It should be noted that the values available in the open literature vary $[25]^{1}$ and depend also on the $\mathrm{Li} / \mathrm{Nb}$ ratio [26]. Values for $\beta$ can be calculated from the temperature-dependent Sellmeier equation; coefficients for $\mathrm{LiNbO}_{3}$ can be found in Refs. [25,27]. Upper limits for the absorption in $\mathrm{LiNbO}_{3}$ crystals are also given in Ref. [27].

Photo refractive damage thresholds for $\mathrm{LiNbO}_{3}$ crystals are usually too low for any application involving high intensity laser beams. However, doping can increase the threshold for the onset of photorefractivity in $\mathrm{LiNbO}_{3}$ crystals by several orders of magnitude [28,29] without changing the electro-optical and thermal material parameters significantly. The most common dopant is magnesium oxide. Vendors quote damage thresholds of $400 \mathrm{~kW} / \mathrm{cm}^{2}$ for their $\mathrm{MgO}: \mathrm{LiNbO}_{3}$ crystals [30]. Thus doped $\mathrm{LiNbO}_{3}$ crystals such as $\mathrm{MgO}: \mathrm{LiNbO}_{3}$ are potential candidates for medium power detectors (20-200 W) although their FOM is still a factor 20 below the FOM of rubidium titanyl phosphate, RTP (described in the next section). The transmittance of doped and undoped $\mathrm{LiNbO}_{3}$ at shorter wavelengths decreases slowly until the UV absorption edge of $\mathrm{LiNbO}_{3}$ near $310 \mathrm{~nm}$ [29]. The increased absorption will limit the potential for applications of even doped $\mathrm{LiNbO}_{3}$ in the visible in interferometric gravitational wave detectors.

\section{The $\mathrm{MTiOXO}_{4}$-family}

The $\mathrm{MTiOXO}_{4}$-family of nonlinear crystals (where the metal $\mathrm{M}$ can be $\mathrm{K}$, $\mathrm{Rb}$, or $\mathrm{Cs}$ and $\mathrm{X}$ can be $\mathrm{P}$ or As) have fairly similar optical properties [31]. They have very low absorption coefficients in the 1 to $2 \mu \mathrm{m}$ region making them ideal for lasers and interferometers working in this wavelength region. The first absorption band is typically around $\lambda=2.8 \mu \mathrm{m}$, likely caused by ionized $\mathrm{O}^{-} \mathrm{H}$-molecules inside the crystal. Additional absorption bands are caused by overtones of vibrational modes in the $\mathrm{XO}_{4}$ tetrahedra starting above $3 \mu \mathrm{m}$ [32]. The UV-absorption edge of all crystals within the $\mathrm{MTiOXO}_{4}$ family is near $350 \mathrm{~nm}$ [32]. Similar to $\mathrm{LiNbO}_{3}$, the largest electro-optical coefficient in all $\mathrm{MTiOXO}_{4}$ crystals is $r_{33}$. The $\mathrm{MTiOXO}_{4}$ crystals are all characterized by a very high damage threshold, including photorefractive damage which greatly exceeds the damage threshold of $\mathrm{LiNbO}_{3}$ [33].

The most common crystal within this family is KTP. It has been commercially available for decades and has been extensively studied. Still, the thermal and thermo-optical parameters available in the literature vary by $\sim 50 \%$ between different sources [3436], possibly due to difference in growth techniques. An absorption coefficient of $\gamma=$ $1.21 \times 10^{-4} / \mathrm{cm}$ was measured using laser calorimetry at $1.08 \mu \mathrm{m}$ [37], although most vendors quote higher absorption coefficients between 6 and $10 \times 10^{-3} / \mathrm{cm}[36,38]$ at $1,064 \mathrm{~nm}$.

An even higher optical damage threshold was reported for RTP [39]. It has also a slightly larger electro-optical parameter and lower electrical loss angle as the mobility of the weakly coupled larger Rb-ions is smaller than the mobility of the weakly

\footnotetext{
1 Some values are within $\sim 20 \%$ of each other and others are vastly different. http://www.hilger-crystals. co.uk/properties.asp?material $=17$.
} 
coupled K-ions [31]. The thermo-optical parameters found in the literature are also not completely consistent [40-44]. The main advantage of RTP is its very low absorption coefficient. Raicol, the vendor which produces the Advanced LIGO RTP crystals, gives an upper limit for the optical absorption of $\gamma<10^{-4} / \mathrm{cm}$ [45].

Scaling measurements of thermal lensing in RTP made at $100 \mathrm{~W}$ powers [44], RTP crystals can safely be used below $1 \mathrm{~kW}$ laser powers at $1,064 \mathrm{~nm}$. Beyond $1 \mathrm{~kW}$ further reductions in the heat-generating absorption of RTP or the use of active thermal compensation or passive thermal correction systems (see Sect. 3 below) might be necessary. $\mathrm{MTiOXO}_{4}$-based modulators can probably be used at similar power levels at $1.5 \mu \mathrm{m}$ as the absorption in RTP (and also in KTP) is expected to be as low if not lower than at $1.064 \mathrm{~nm}$ [45]. However, detailed thermal lensing and mode deformation measurements for any of these crystals at high power levels at this wavelength have not yet been performed to our knowledge.

The other isomorphs of the $\mathrm{MTiOXO}_{4}$-family such as KTA and RTA have also fairly high optical damage thresholds and similar electro-optical, thermal, and thermooptical parameters when compared to KTP and RTP [31]. [46] quotes an absorption coefficient of $<500 \mathrm{ppm} / \mathrm{cm}$ for KTA identical to their absorption coefficient for RTP. The absorption of all crystals in the $\mathrm{MTiOXO}_{4}$-family appears to be small enough such that they can potentially be used between 1 and $1.5 \mu \mathrm{m}$ possibly up to $1 \mathrm{~kW}$ power levels.

Like most materials, optical absorption in the $\mathrm{MTiOXO}_{4}$-family increases when the wavelength is reduced. Raicol measured absorption values of $2-4 \times 10^{-3} / \mathrm{cm}$ at $532 \mathrm{~nm}$ for their RTP crystals [45]. This would limit the laser power to $\sim 50 \mathrm{~W}$ for gravitational wave detectors assuming the same crystal length.

\section{$B B O$}

For wavelengths in the visible and near-UV regions, $\beta$-barium borate $(\mathrm{BBO})$ and its derivatives are often used. BBO is uniaxial and has a very high damage threshold. Values larger than $3 \mathrm{~kW} / \mathrm{cm}^{2}$ for $\mathrm{cw}$-light have been quoted by multiple vendors [47]. BBO has a negative thermo-optical coefficient which prevents self-focusing. Its UV-absorption edge is around $190 \mathrm{~nm}$. The main disadvantage is that its largest electro-optical coefficient $r_{11}$ is small compared to the other crystals. Unfortunately, thermal and thermo-optic crystal parameters also vary in the literature. Sources quote a factor of 10 difference in the thermal conductivities in the two directions parallel to the beam propagation direction $[35,48]$, which would cause substantial asymmetric thermal lensing at kilowatt powers. However, other vendors quote larger and fairly identical conductivities [49]. [48] gives an absorption coefficient of $\gamma<0.1 \% / \mathrm{cm}$ for $\lambda=1.064 \mu \mathrm{m}$. Optical absorption at shorter wavelength is also increasing to $1 \% / \mathrm{cm}$ at $\lambda=532 \mathrm{~nm}$ [26]. Based on this information, RTP appears to have a larger FOM at $\lambda=532 \mathrm{~nm}$ than BBO mainly due to its much higher electro-optical coefficient. Only at wavelengths near and obviously below the UV absorption edge of RTP will BBO have a better figure of merit.

Obviously, there are many more non-linear crystals commercially available and several promising types that are not commercially available yet. For the near infrared region, RTP and potentially its isomorphs KTP, KTA, RTA, or even CTA may 
present a very good choice for power levels maybe even beyond $1 \mathrm{~kW}$. And as noted previously, $\mathrm{MgO}: \mathrm{LiNbO}_{3}$ might be a potential alternative at kilowatt powers. The key parameter for all these crystals is the absorption coefficient. At shorter wavelengths, the absorption in RTP and $\mathrm{MgO}: \mathrm{LiNbO}_{3}$ increases rapidly and will limit their use to fairly low power levels, perhaps in the $50 \mathrm{~W}$ range. At wavelengths below $450 \mathrm{~nm}$, BBO might be a better choice although its relatively low electro-optical coefficient would reduce the possible modulation indices significantly. While several good candidate materials have been identified, extensive experimental tests will be necessary to develop electro-optical modulators in the near infrared for laser powers exceeding $\sim 1 \mathrm{~kW}$. More difficult will be the development of electro-optical modulators for the visible range which can operate at laser power levels exceeding $100 \mathrm{~W}$. All these power levels can potentially be further increased using thermal compensation techniques. These have been developed for Faraday rotators and will be described in the next section.

\subsection{Faraday rotators}

Traditional Faraday rotators make use of the Faraday effect to induce a non-reciprocal linear polarization rotation which depends on the direction of the applied magnetic field $B$ relative to the axis of the crystal. The polarization rotation angle is given by $\theta=B V L_{\text {optical }}$ where $V$ is the Verdet constant and $L_{\text {optical }}$ is the optical path length.

Ideally, Faraday isolators should have high isolation ratios $(>30 \mathrm{~dB})$ that are independent of input power, high forward-going and backward-going transmission (approaching 100\%), minimal thermal lensing and thermal beam deflection. However, spatially non-uniform fields $B=B(\mathbf{r})$ as well as the temperature and spatial dependence of the Verdet constant $V=V(T(\mathbf{r}))$ and optical path $L_{\text {optical }}=$ $L_{\text {physical }} \cdot n(T(\mathbf{r}))$ compromise the performance of Faraday isolators with respect to all of these functions.

\section{Materials for 1,064 nm operation: TGG, GGG, YAG}

Faraday isolators in current and second generation detectors operating at 1,064 nm are based on terbium gallium garnet (TGG) magneto-optical crystals. Isolation ratios approaching $50 \mathrm{~dB}$ (power) have been demonstrated with laser powers of $200 \mathrm{~W}$ without significant degradation of the spatial laser mode [50]. To achieve this performance, high average power Faraday isolators make use of a dual crystal compensated Faraday rotators [51] and negative $d n / d T$ thermal lens compensating elements [52] to increase the isolation ratio and minimize thermally induced modal distortions.

A figure of merit for the power handling capabilities of magneto-optic crystals and glasses can be defined as $\mu \equiv V \kappa / \gamma Q$ where $Q$ is the thermo-optical constant (related to the photo-elastic strain tensor) [51]. Larger Verdet constants result in shorter crystal lengths and less absorption. Similarly, reducing the intrinsic optical absorption minimizes the temperature rise in the magneto-optic material. High thermal conductivity leads to smaller temperature gradients, improving thermal self-focusing. Large thermo-optical constants couple to the temperature gradient and lead to both linear and 
circular birefringence within the medium resulting in inhomogeneous polarization in the transmitted beam.

There are several routes to scaling TGG to kilowatt-class average power operation in the near infrared, including i) the use of thin-disk geometries, ii) cryogenic cooling of the magneto-optical material, iii) designing higher compact magnetic fields, and iv) the use of novel magneto-optical materials. The use of thin-disk geometries similar to that used in high power lasers [53] instead of currently employed rod geometries can significantly reduce transverse temperature gradients. Theoretical studies have predicted and experiments have subsequently confirmed that the thermally induced depolarization is proportional to $\left(D_{\text {disk }} / L_{\text {disk }}\right)^{-4}$, where $D_{\text {disk }}$ and $L_{\text {disk }}$ are the disk diameter and length [54].

Cryogenic cooling of magneto-optical materials results in a large increase in $V$ and improved thermo-optical parameters and is another possible route. Experiments have shown that reducing the temperature of TGG to $77 \mathrm{~K}$ results in a fourfold increase in the Verdet constant [from $40 \mathrm{rad} /(\mathrm{T} \mathrm{m})$ to $160 \mathrm{rad} /(\mathrm{T} \mathrm{m})$ ] [55], a factor of 5 reduction in $L_{\text {physical }}$, and a factor of 3 reduction of the product $\gamma Q$ [56,57]. In flux-grown TGG crystals, cooling to $77 \mathrm{~K}$ also increases $\kappa$ twofold. (Remarkably, operation at liquid He temperatures produces an $87 \times$ increase in $V$ over its room temperature value [55]).

Increasing the magnetic field strength $B$ proportionally reduces the physical path length $L_{\text {physical }}$ needed for a desired rotation angle. Thus another approach is to utilize compact, high field magnets for Faraday rotators. Mukhin et al. have demonstrated the operation of a Faraday isolator using a specially designed 2.1 T magnet capable of $30 \mathrm{~dB}$ isolation for $330 \mathrm{~W}$ incident powers using a single [001] orientation TGG crystal [58], however the design of the magnet configuration is complicated and cumbersome to implement.

An alternative approach to high power operation is the use of other magneto-optical crystals with lower absorption and higher thermal conductivities. Examples include Yttrium Aluminum Garnet (YAG) and gadolinium gallium oxide (GGG). Absorption in YAG in the near infrared is $0.0015 / \mathrm{cm}$, approximately four times less than TGG absorption (which can vary depending on supplier). GGG is also reported to have low absorption in the 0.3-7 $\mu \mathrm{m}$ spectral window [59]. Although the Verdet constant of GGG is 6 times lower than in TGG $(6.5 \mathrm{rad} /(\mathrm{T} \mathrm{m}))$ - the thermal conductivity $\kappa$ in GGG $(9 \mathrm{~W} / \mathrm{K} \mathrm{m})$ is twice as large as TGG, and when cooled to $70 \mathrm{~K}, \kappa$ increases by a factor of approximately 7 . Because GGG is paramagnetic, its Verdet constant changes inversely with temperature. Thus, cooling of a GGG magneto-optical element to $77 \mathrm{~K}$ will provide a 2.3 -fold increase in maximal power with the isolation ratio being unchanged. The same calculation for Nd:YAG results a fourfold increase in laser power at nitrogen temperatures. Another possibility is the use of TGG ceramics, which have been shown to have the Verdet constants essentially identical to crystalline TGG at all temperatures [55]).

\section{Materials for 1.3-1.6 $\mu \mathrm{m}$ : rare earth iron garnets and derivatives}

For detectors operating at longer wavelengths, increased absorption in TGG due to $\mathrm{Tb}^{3+}$ makes these materials unlikely candidates for Faraday isolators. Fortunately, the $1.3-1.6 \mu \mathrm{m}$ wavelength range benefits from significant technology development 
related to the telecommunications sector. The development of Faraday isolators in this range has been primarily centered around rare earth iron garnets (RIGs) and their derivatives [60]. Original efforts centered on yttrium iron garnet $\left(\mathrm{Y}_{3} \mathrm{Fe}_{5} \mathrm{O}_{12}\right.$ or (YIG) but more recently $\{\mathrm{BiRE}\}_{3}(\mathrm{FeGaAl})_{5} \mathrm{O}_{12}$ have been used because of their much greater intrinsic Faraday rotation. Unlike paramagnetic magneto-optic materials (eg., TGG) in which saturated magnetization is achieved using an external magnetic field, the doped RIGs are ferrimagnetic; individual domains in the films are grown in a saturated state. The net Faraday rotation arises from the integrated contributions of all domains in the material. Thus, RIGs have the advantage that external magnetic fields are not needed.

RIG materials are typically grown in thin films via liquid phase epitaxy on latticematched substrates. GGG is typically used as the substrate for most RIG films, although bismuth-substituted RIG (Bi:RIG) is grown on calcium magnesium zirconium-substituted GGG for optimal lattice matching. For operation using large mode sizes, the laser beam impinges on the face film and is transmitted through the substrate. Typical films thicknesses needed for $45^{\circ}$ rotation are in the range $300-500 \mu \mathrm{m}$.

Absorption in RIG is typically in the $1-10 / \mathrm{cm}$ range in $1,300-1,550 \mathrm{~nm}$ range $[60,61]$, corresponding to an internal transmission in the range of 30-90\%. Currently, these levels of absorption would prove very problematic at high laser powers. Excess absorption is dominated by the lack of stoichiometry of garnet, although precise control of growth conditions can improve the absorption characteristics [62]. Internal transmissions in excess of $99 \%$ have been achieved for Bi:RIG materials, comparable to that obtained with TGG at $1,064 \mathrm{~nm}$. Isolation ratios as high as $37 \mathrm{~dB}$ have been achieved using YIG in a waveguide geometry on a GGG substrate [63], but are typically less than $30 \mathrm{~dB}$ for thick film conventional geometries. All RIG-based materials exhibit wavelength-dependent Faraday rotation, problematic for telecommunications applications but not for gravitational wave detectors which operate at single wavelengths. Optical birefringence is inherent in thin film RIG-based materials, arising from anisotropies in the growth of the film (RIG films are uniaxial), from stress induced by lattice-mismatch between the film and the substrate, and via shapebirefringence inherent in thin film geometries. Anisotropies and lattice mismatch can be controlled by careful growth techniques.

The best prospects for high power operation seem to lie with Bi-doped RIG materials not employing Tb or Er, which both contribute to increased absorption in the 1,300$1,600 \mathrm{~nm}$ range. However, not much is known concerning high power operation of RIG-based Faraday isolators. Reasonably large $(11 \mathrm{~mm})$ aperture RIG isolators have been demonstrated at $10 \mathrm{~W}$ power levels [62]. The magnitude of the polarization rotation angle typically increases as the temperature is decreased, i.e., $d \theta_{\text {rot }} / d T<0$. As with paramagnetic crystals and glasses, decreasing the operating temperature results in shorter path lengths for a specific rotation angle. Temperature derivatives have been measured for many RIG materials at $T=300 \mathrm{~K}[60,64,65]$, but not at cryogenic temperatures to our knowledge. In addition, absorption can decrease as the temperature decreases due to shifts in the absorption edge of the material provided mid-infrared absorption bands lie far away from the desired operation wavelength. Finally, differential thermal expansion between the lattice and substrate can lead to increased stress birefringence and degradation of the wavefront via film 'bowing'. 


\section{Materials for short wavelength operation}

The prospects for designing short wavelength Faraday isolators are considerably less obvious at the present time. Optical absorption is more severe at short wavelengths, and thermal effects are correspondingly more pronounced. Absorption in TGG begins to rise at wavelengths below $900 \mathrm{~nm}$, with $\gamma=0.15 / \mathrm{cm}$ at $532 \mathrm{~nm}$ (30 times greater than at $1,064 \mathrm{~nm}$ ). The absorption edge of TGG occurs at $390 \mathrm{~nm}$ [62]. Faraday isolators using potassium dihydrogen phosphate (KDP) isomorphs have been developed for ultraviolet wavelengths [66]. KDP and its isomorphs (DKDP, ADA, ADP, DKDA) have room temperature Verdet constants in range $12.1-27.3 \mathrm{rad} /(\mathrm{T} \mathrm{m})$ at $351 \mathrm{~nm}$, slightly less than TGG. The absorption edge occurs in the $200-300 \mathrm{~nm}$ range is approximately depending on isomorph. Residual absorption at a level of $\gamma<0.01 / \mathrm{cm}$ has been observed to $350 \mathrm{~nm}$. In addition, Nd-doped fluorozirconate glasses have been shown to exhibit the Faraday effect [67], however there are no commercial sources available for this material, thus a significant material development effort would be needed.

\section{Vacuum operation of faraday isolators}

Operation in vacuum requires special considerations - convective cooling at the faces of the optical components is no longer an effective heat removal channel and efficient heat sinking is essential to minimize thermal lensing and depolarization. Faraday isolators carefully aligned in air can experience a dramatic reduction in isolation ratio $(>10-15 \mathrm{~dB})$ when placed in vacuum [68]. In addition, long term operation of the Faraday isolators in LIGO have shown that the rejected beam experiences a deflection as the input power changes [69]. Because the rejected beam is sensed for both length and alignment control in the interferometer, thermal steering leads to offsets in the length and alignment controls. Minimization of this drift is important for future GW interferometers. Experiments have shown that for powers up to $100 \mathrm{~W}$, most of the effects come from thermal loading in the polarizers. The use of commercial thin film polarizers (TFPs) results in a 50-100 fold reduction in the angular steering of the beam over alternative polarizers, albeit at the expense of the isolation ratio. For kilowatt operation at long wavelengths, polarizers made of ultralow loss fused silica $(<10 \mathrm{ppm} / \mathrm{cm}$ absorption losses at $1,064 \mathrm{~nm})$ will be required with optical coatings that have less than $2-3$ ppm absorption.

\section{Nano-structured optics}

Thermal noise of test mass mirrors will be one of the dominating noise sources in future gravitational wave detectors. Thermal noise arises from thermally driven motions of test mass mirror surfaces and thermally driven changes of optical path lengths through mirrors. Cryogenic cooling is a straightforward approach to reduce thermal noise. However, nano-structured optical components have the potential to significantly support the effort of thermal noise reduction, at room temperature as well as cryogenic temperatures. Here, we consider two different approaches of how nano-structured optics might be useful in future gravitational wave detectors. 


\section{All-reflective gratings}

Gravitational wave detectors currently use not only reflective optics but also transmissive optics, for example as beam splitters and cavity couplers. In transmissive optics thermal noise arises from statistical temperature fluctuations and the temperature dependent refractive index. A significant problem affecting transmissive optics is the absorption of laser light which result in additional local temperature variations, and may also result in heating. The latter effect is expected to be substantial for future gravitational wave detectors with cooled test masses since high arm cavity circulating laser powers $(\sim 1 \mathrm{MW})$ will be used. In recent table-top experiments, building on earlier work by Sun and Byer [70], it was demonstrated that diffractive grating structures on mirror surfaces can be used to realize high-quality all-reflective beam splitters and cavity couplers.

An all-reflective alternative to a transmissive 50/50 beam-splitter can be realized by a dielectric reflection grating with two diffraction orders (0th and 1st) that splits the incoming light field in two equal parts (see Fig. 1a). Since for the 1st order diffracted beam the angle of incidence and the diffraction angle are not equal, the beam is deformed in horizontal direction. Despite this additional complication it is possible to build an all-reflective Michelson interferometer with a very high fringe contrast (>0.9997) based on diffractive beam-splitters [71].

Conventional Fabry-Perot cavities require a partly transmissive mirror to couple light into the resonator. Two different concepts have been demonstrated that allow for an all-reflective coupling of Fabry-Perot resonators. First, a grating in a 1st order Littrow arrangement can be used. Here, the 1st order diffracted beam counter propagates the input beam (see Fig. 1b) and the specular 0th order is used to couple light into the cavity. In this configuration the finesse of the cavity is limited by the 1st order diffraction efficiency of the grating. The first demonstration was done in [70] where a cavity finesse of 53 was achieved. More recently, finesse values of up to $\approx 1,450$ have been demonstrated [72]. The second concepts uses a so-called 2nd order Littrow arrangement. Here, the 1st diffraction order is used to couple light into the cavity and the 2 nd order is sent towards the laser input (Fig. 1c). In this configuration the finesse of the resulting Fabry-Perot cavity is limited by the specular reflectivity of the grating for normal incidence. Therefore a grating that unites low diffraction efficiency and a high reflectivity for normal incidence is optimal to realize a high finesse cavity. Unlike a partly transmissive mirror this coupler splits the incoming light field not
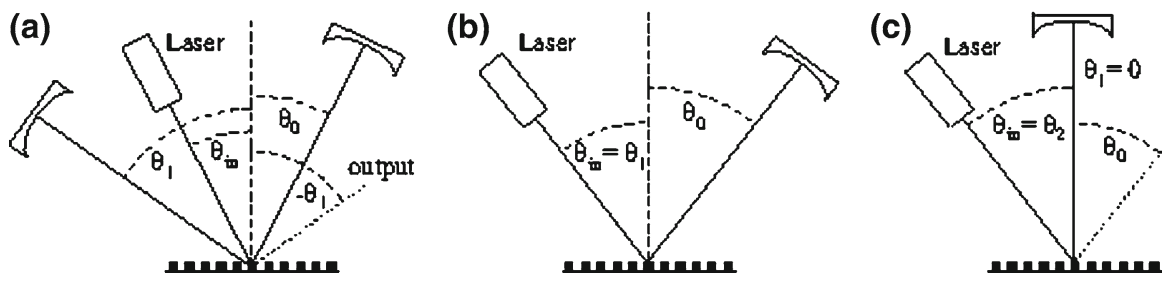

Fig. 1 a Reflection grating with two existing orders can be used as a 50/50 beam splitter in a Michelson interferometer; b Grating coupled Fabry-Perot cavity in 1st order Littrow mount; c 2nd order Littrow mount 
in two but in three partial beams. This additional port leads to more complex phase relations between the fields yielding different cavity properties and allow for the realization of completely new interferometer concepts $[73,74]$. The concept of coupling to a cavity with such 3-port devices was successfully demonstrated with a cavity finesse of 400 [75].

In comparison to a standard reflective component (mirror or beam splitter) the diffractive nature of the gratings causes an additional coupling of geometry changes into alignment and phase noise [76]. Therefore different read-out techniques [77] and interferometer topologies are currently under investigation in order to reduce the coupling of this noise source.

\section{Waveguide mirrors}

The dominant thermal noise source in current gravitational wave detectors comes from dielectric mirror coatings[78]. Conventional schemes for high reflectivity (HR) mirrors require up to 40 layers of $\mathrm{Ta}_{2} \mathrm{O}_{5}$ and $\mathrm{SiO}_{2}$ each having an optical thickness of a quarter wavelength. The coating thermal noise is due to the mechanical loss of the layers with a dominant contribution of $\mathrm{Ta}_{2} \mathrm{O}_{5}$. One approach to this problem is to design alternative multilayer stacks deviating from the classical quarter wavelength design aiming for less $\mathrm{Ta}_{2} \mathrm{O}_{5}$ [79]. Also doping $\mathrm{Ta}_{2} \mathrm{O}_{5}$ with $\mathrm{TiO}_{2}$ has been investigated with an achieved reduction of the mechanical loss angle of 1.5 [80].

Concepts avoiding HR-multilayer stacks but providing high reflectivity have been proposed, namely corner reflectors [81], coating-free mirrors [82]. Both are based on total internal reflection, which is connected with additional optical paths inside substrates, which give rise to absorption and increased thermo-refractive noise resulting from a temperature dependent refractive index.

Another possibility of avoiding multilayer stacks are nanostructured waveguide layers. Single layer as well as monolithic grating waveguide structures have been proposed $[83,84]$ as low thermal noise alternative to conventional multilayer stacks for high reflectivity test mass mirrors in future gravitational wave detectors. The principle is shown in Fig. 2 using the ray picture [85].

The nanostructured surface can be designed in order to allow for the 0th order in reflection and 0 th and \pm 1 st orders in transmission. The \pm 1 st orders are guided in
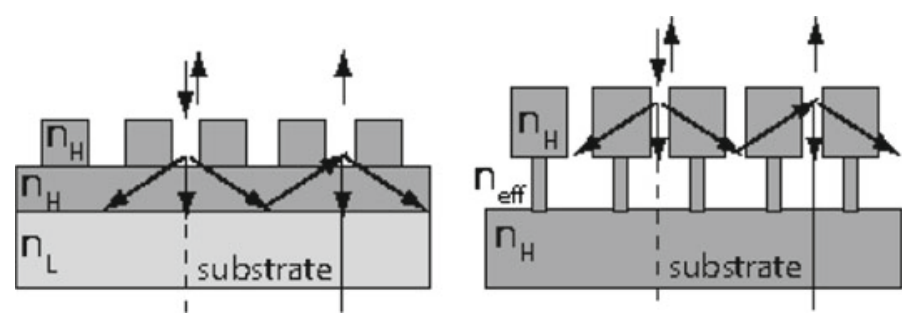

Fig. 2 Principle of single layer (left) and monolithic (right) waveguide mirrors in the ray picture. The incoupled \pm 1 st transmitted orders are totaly reflected at the substrate or thin ridges, respectively, and coupled out at the grating structure. For proper chosen dimensions of the nanostructured surface and waveguide one can achieve constructive interference and thus $100 \%$ reflectivity 

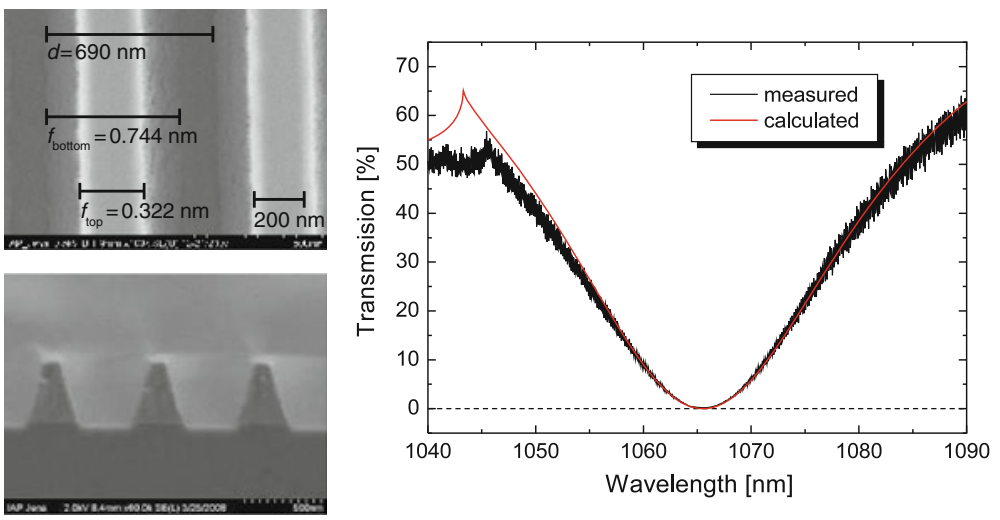

Fig. 3 Top (top left) and cross-sectional view (bottom left) of a fabricated waveguide mirror. Right spectral transmittance under normal incidence of a fabricated waveguide mirror that had reflectivity of $>99 \%$ at a wavelength of $1,064 \mathrm{~nm}$, [87]

a waveguide layer and partly coupled out. The waveguide mirror dimensions can be adjusted to achieve constructive interference of the outcoupled and specular reflected light and thus $100 \%$ reflectivity. The exact optical properties of such a device can be found with rigorous coupled wave analysis (RCWA) [86]. We experimentally realized grating waveguide structures and demonstrated a reflectivity $>99 \%$ in a cavity setup[87], which was in good agreement with RCWA calculations (see Fig. 3).

Further design optimizations and precise characterization of the optical and mechanical properties of microstructured surfaces are of great interest. Higher order beams are under discussion for reducing coating thermal noise [88]. Since this seems to be compatible with gratings, one could in principle benefit from both techniques.

To conclude, currently used lithography techniques allow for large area structures, but are limited to substrate thicknesses of a few millimeters, which is far below the values that are required in gravitational wave detection. One idea to fabricate massive nanostructured test masses is to use bonding techniques to connect a structured wafer with a thick substrate, which in principle could provide a high mechanical quality factor for the entire component.

\section{Squeezed light}

Squeezed states of light belong to the class of so-called nonclassical states of light [89]. Quantum noise limited laser light occurs in a coherent state, where the independent arrival times of photons leads to Poisson counting statistics. In contrast with coherent states, for squeezed states the detection events of photons are not independent of one another, but rather show quantum correlations. These correlations can be used to squeeze the interferometer output below the shot noise limit. Caves first proposed that injection of squeezed states of light into the output port of an interferometer operated close to a dark fringe can be used to improve the measurement sensitivity [3]. Caves envisioned injection of squeezed states as a way of increasing the detector 
sensitivity beyond the shot noise limit of the most powerful lasers available at the time. Three decades later, with approximately 100 times more laser power available, but with significant technical challenges to maintaining high circulating power in the interferometer, squeezed states allow for improvement of the quantum-noise-limited sensitivity without further increasing the circulating laser power. High circulating laser power, and concomitant residual absorption in the test masses, poses a serious challenge for future detectors with cryogenically cooled test mass, in particular. The same quantum fluctuations of the light that give rise to shot noise also introduce radiation pressure (back-action) noise on the test masses. Radiation pressure noise dominates at low detection frequencies, while shot noise dominates at higher frequencies, and when these two noises are equal (for a given power level), we get the standard quantum limit (SQL). In 1990 Jaeckel and Renault [90] showed that squeezed states can be used to simultaneously reduce the shot noise and radiation pressure noise, and that the SQL of a test mass position measurement can be beaten. Measurement sensitivity beyond the SQL corresponds to a quantum non-demolition (QND) measurement $[91,92]$. Practically a broadband nonclassical noise reduction beyond the SQL can be achieved if the injection of squeezed light is combined with additional narrowband optical filter cavities at the interferometer dark port $[92,93]$. However, the realization of such filter cavities is technically challenging. In all experiments to date the quantum radiation pressure noise regime has been dominated by technical noise and thermal noise, so squeezed state injection will first be used to exclusively reduce the shot noise in gravitational wave detectors.

A gravitational wave signal at frequency $f$ appears as a modulation of the (weak) interferometer output field. The strength of this modulation signal and its (quantum) noise can be described by two quadrature operators $\hat{X}_{1,2}(f, \Delta f, t)$. Here, the subscripts 1 and 2 correspond to the non-commuting amplitude and phase quadratures, respectively, and $\Delta f$ to the resolution bandwidth (RBW). For coherent states the variances of the quadrature operators are identical and typically normalized to unity. With this normalization, the Heisenberg uncertainty relation (HUR) sets the following lower bound for the product of the quadrature variances for both coherent states, and for vacuum states:

$$
\Delta^{2} \hat{X}_{1}(f, \Delta f, t) \cdot \Delta^{2} \hat{X}_{2}(f, \Delta f, t) \geq 1
$$

According to Caves [3], the quantum noise at the output of the interferometer is due to the beat between the (weak) coherent signal field and the vacuum field. Squeezed vacuum states can be injected into the output port to improve the interferometer sensitivity beyond the shot noise limit, provided the squeezed quadrature is adjusted to the quadrature of the signal modulation field being detected. In case of an interferometer with carrier tuned signal-recycling and homodyne (DC) read out, for example, this would be the amplitude quadrature of the interferometer's output field. In this case we simply require a broadband amplitude squeezed field where $\Delta^{2} \hat{X}_{1}(f, \Delta f, t)$ is always below the unity vacuum noise reference for all sideband frequencies $f$ within the detection band. 


\section{Generation of squeezed light}

The first observation of squeezed states was by Slusher et al. [94] in 1985. Since then different techniques for the generation of squeezed light have evolved. A schematic setup for one of the most successful approaches to squeezed light generation-optical parametric oscillation (OPO) - is shown in Fig. 4. A second-order nonlinear material like $\mathrm{MgO}: \mathrm{LiNbO}_{3}$ or periodically poled potassium titanyl phosphate (PPKTP) can be used to produce broadband squeezing at the carrier wavelength of present day gravitational wave detectors $(1,064 \mathrm{~nm})$. In order to do so a continuous-wave second harmonic light field (green light at $532 \mathrm{~nm}$ ) is focussed into the nonlinear crystal, which is placed inside a cavity [95]. Multiple passes in the cavity increases the strength of the parametric process, i.e., the parametric down conversion of green photons into correlated pairs of infrared photons. The quantum correlations lead to production of squeezed states at $1,064 \mathrm{~nm}$. The squeezed states can be detected by balanced homodyne detection, where the squeezed field is overlapped with an optical local oscillator field on a 50\%/50\% beam splitter (Fig. 4). Note that for squeezed light injection into an interferometer, the local oscillator may also be provided by the weak laser field exiting the interferometer's antisymmetric port.

In recent years, several table-top experiments at the Australian National University in Canberra [96], and at the Albert-Einstein-Institute in Hannover [97-99], along with a test of injecting squeezed states into a suspended-mirror prototype interferometer, carried out at MIT and Caltech [100], have paved the way for a first application of squeezed light in gravitational wave detectors. These experiments together have demonstrated (i) that the shot noise reduction through squeezed states is compatible with a variety of interferometer topologies [100,101]; (ii) that high levels of squeezing can efficiently be produced; and (iii) that they can be stably controlled $[97,98]$

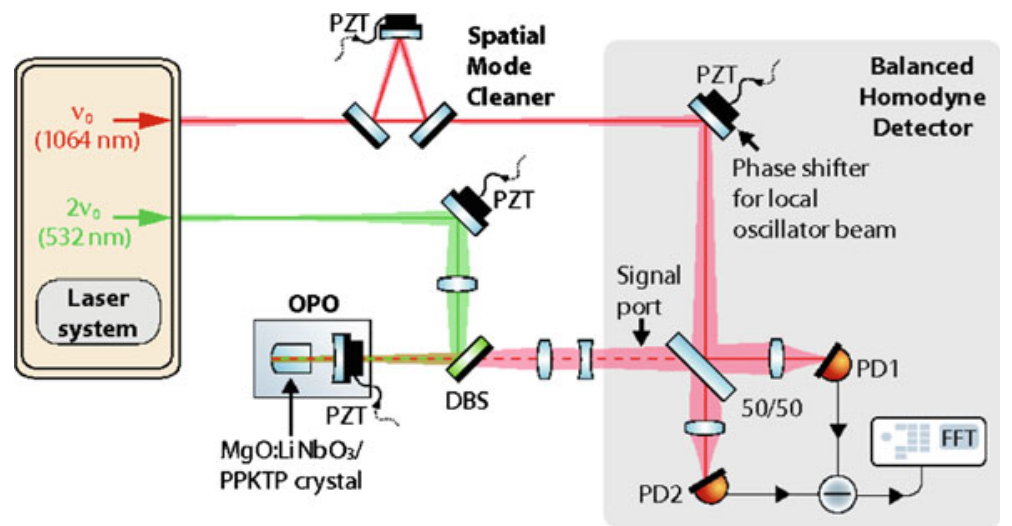

Fig. 4 Generation and detection of squeezed light at 1,064 nm. A second-order nonlinear crystal made from $\mathrm{MgO}: \mathrm{LiNbO}_{3}$ or PPKTP is pumped with continuous wave light at $532 \mathrm{~nm}$. Optical parametric oscillation (OPO), also called parametric down-conversion, produces a squeezed quantum noise of the reflected (vacuum) mode of light at $1,064 \mathrm{~nm}$. $D B S$ dichroic beam splitter, $P D$ photo diode, $P Z T$ piezo-electric transducer for positioning of mirrors 
for application in the detection band of gravitational wave detectors with suspended mirror dynamics [100].

The strongest squeezing observed so far provides a nonclassically reduced shot noise by a factor of ten $(10 \mathrm{~dB})$ [99]. Injected into the output port of an interferometer, this level of squeezing increases the interferometer sensitivity by exactly the same factor as an equivalent increase in laser power. Note, that optical power (photon) loss $\left(t^{2}\right)$ increases the value for the squeezed variance according to $\Delta^{2} \hat{X}^{\prime}=\Delta^{2} \hat{X}\left(1-t^{2}\right)+t^{2}$. For future gravitational wave detectors the reduction of optical loss will, therefore, be increasingly important.

\section{Squeezing light at audio frequencies}

A necessary requirement for the application of squeezed states of light in gravitational wave detectors is the ability to generate squeezed states at audio-band Fourier frequencies. The first such demonstration was achieved in 2004 by McKenzie et al. [96] where a broadband squeezed field down to a few hundreds of Hertz was generated. In an improved experiment in 2007, Vahlbruch et al. [101] observed squeezing down to $1 \mathrm{~Hz}$, as shown in Fig. 5. Scattered and frequency shifted photons were identified to be a major noise source at low frequencies in previous and also current experiments with squeezed light.

Gravitational wave detectors require a long-term stable phase control of the injected squeezed states in relation to the interferometer's main laser field. This requirement is in particular difficult to realize for squeezing at low frequencies, because coherent control fields may introduce classical noise. A highly stable coherent control scheme was demonstrated in $[97,98]$. It relies on a coherent control field that is frequency shifted by several megahertz with respect to the squeezed field and is also parametrically

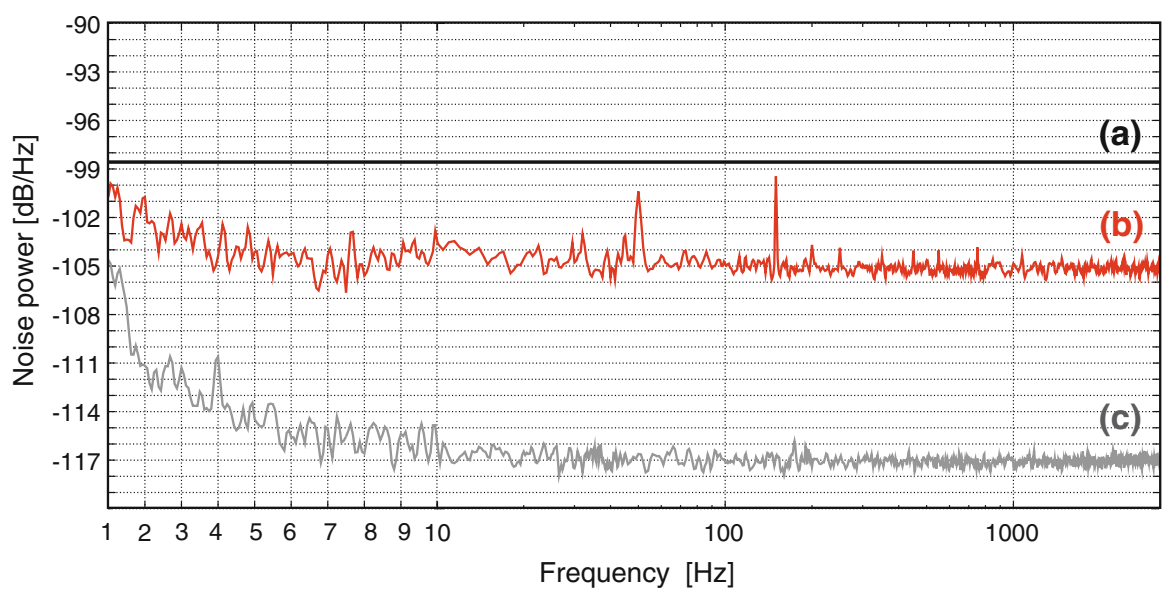

Fig. 5 Trace a shows the (theoretical) vacuum noise level of our homodyne detector. Trace b shows the noise powers of squeezed states measured with the same detector setting. Trace c shows the electronic (dark) noise floor. Squeezed vacuum noise was observed throughout the complete spectrum from $1 \mathrm{~Hz}$ to above $3 \mathrm{kHz}$. A nonclassical noise suppression of up to $6.5 \mathrm{~dB}$ below vacuum noise was observed here [101] 
amplified by the squeezed light source, thereby giving a phase reference for the parametric process.

An alternative approach, termed quantum noise locking [102], was used in the 2004 ANU experiment [96]. In this technique, the asymmetry in the quadrature variances is directly used to obtain a phase-sensitive readout and to lock the phase of the squeezed vacuum state. Whilst not as stable as coherent control schemes, it is adequate for moderately squeezed states and is simple to implement.

\section{Compatibility of squeezed light injection with detector topologies}

To gain significant and broadband sensitivity improvement when a broadband amplitude squeezed field is injected into the interferometer's output port, (ideally) lossless reflection of the squeezed states without frequency-dependent rotations in quadrature phase space must be ensured. However, gravitational wave detectors are not just Michelson interferometers, but involve dispersive optical cavities and nonperfect optical components. In [103] it was experimentally shown that the technique of power-recycling is not only compatible with squeezed light injection but also mitigates losses associated with an imperfect dark fringe. In [104] a squeezed light enhanced power-recycled and detuned signal-recycling interferometer was realized. It was successfully demonstrated that a detuned filter cavity in the interferometer's dark port can compensate for the dispersion of the detuned signal recycling cavity. Goda et al. injected squeezed states into the output port of the $40 \mathrm{~m}$ prototype gravitational wave detector at Caltech, and showed that a system consisting of a squeezed light source and an interferometer with suspended mirror can be sufficiently controlled to realize nonclassical sensitivity improvement [100].

The tremendous progress on the generation and control of squeezed states of light made in recent years has demonstrated that this technology is now mature (see also [105]). It is likely that squeezed light injection will play a central role in achieving the sensitivity improvements envisioned for the third generation.

\section{Conclusion}

The enhanced performance of third generation gravitational wave interferometers will rely on a variety of advances in laser sources and optical technologies. Kilowatt-class lasers, electro-optic modulators, and Faraday isolators potentially operating at wavelengths shorter (in the UV or visible spectral regions) or longer (in the near infrared region out to $1.6 \mu \mathrm{m}$ ) will be needed as sources, coupled with techniques for squeezing of the laser field to improve shot noise sensitivity. The development of novel interferometer optics including diffractive optics and waveguide high reflectors will improve the power handling capability and reduce thermal noise.

Acknowledgments The authors would like to thank Vincent Fratello for useful discussions concerning materials for long and short wavelength Faraday isolators. G.M and D.H.R gratefully acknowledge the support of the National Science Foundation (PHY0555453, PHY0757968, and PHY0653582) and R.S. and B.W. the support of the Deutsche Forschungsgemeinschaft (SFB 407, SFB TR7 and QUEST). 
Open Access This article is distributed under the terms of the Creative Commons Attribution Noncommercial License which permits any noncommercial use, distribution, and reproduction in any medium, provided the original author(s) and source are credited.

\section{References}

1. Abbott, R., Adhikari, R., Ballmer, S., Barsotti, L., Evans, M., Fritschel, P., Frolov, V., Mueller, G., Slagmolen, B., Waldman, S.: LIGO-T070247-01-1: AdvLIGO Interferometer Sensing and Control Conceptual Design, Ligo Document Center, https://dcc.ligo.org/ (2007)

2. Conforto, G., DeSalvo, R.: Proposal for lower frequency companions for the advanced LIGO Gravitational Wave Interferometric Detectors. Nuc. Instr. Meth. Phys. Res. A 518, 228 (2004)

3. Caves, C.M.: Phys. Rev. D 23, 1693 (1981)

4. Meers, B.J.: Recycling in laser-interferometric gravitational-wave detectors. Phys. Rev. D 38, 2317 (1988)

5. Rowan, S., et al.: Challenges in thermal noise. Gen. Relativ. Gravit. (this issue) (2010)

6. Mitigating noise in the $1-10 \mathrm{~Hz}$ band, in this issue

7. Willke, B., Danzmann, K., Frede, M., King, P., Kracht, D., Kwee, P., Puncken, O., Savage, J.R.L., Schulz, B., Seifert, F., Veltkamp, C., Wagner, S., Wessels, P., Winkelmann, L.: Stabilized lasers for advanced gravitational wave detectors. Class. Quantum Gravit. 25, 114040 (2008)

8. Frede, M., Wilhelm, R., Kracht, D., Fallnich, C.: Nd:YAG ring laser with $213 \mathrm{~W}$ linearly polarized fundamental mode output power. Opt. Express 13, 7516 (2005)

9. Kwee, P., Willke, B., Danzmann, K.: Shot noise limited laser power stabilization with a high power photodiode array. Opt. Lett. 34(19), 2912 (2009)

10. Sun, K.-X., Fejer, M.M., Gustafson, E., Byer, R.L.: Sagnac interferometer for gravitational-wave detection. Phys. Rev. Lett. 76, 3053 (1996)

11. Chelkowski, et al.: Prospects of higher-order Laguerre-Gauss modes in future gravitational wave detectors. Phys. Rev. D 79, 122002 (2009)

12. Kane, T., Eckardt, R., Byer, R.: Reduced thermal focusing and birefringence in zig-zag slab geometry crystalline lasers. IEEE J. Quantum Electron. 19, 1351 (1983)

13. Tulloch, W.M., Rutherford, T.S., Gustafson, E.K., Byer, R.L.: CW high-power conduction-cooled edge-pumped slab laser, Solid State Lasers VIII 3613, ed. Richard Scheps, SPIE 2 (1999)

14. Rutherford, T.S., Tulloch, W.M., Sinha, S., Byer, R.L.: Yb:YAG and Nd:YAG edge-pumped slab lasers. Opt. Lett. 26, 986 (2001)

15. Bedö, S., Lüthy, W., Weber, H.P.: The effective absorption coefficient in double-clad fibres. Opt. Commun. 99, 331 (1993)

16. Limpert, J., Roser, F., Klingebiel, S., Schreiber, T., Wirth, C., Peschel, T., Eberhardt, R., Tünnermann, A.: The rising power of fiber lasers and amplifiers, Selected Topics in Quantum Electronics. IEEE J. 13, 537 (2007)

17. Jeong, Y., Sahu, J., Payne, D., Nilsson, J.: Ytterbium-doped large-core fiber laser with $1.36 \mathrm{kw}$ continuous-wave output power. Opt. Express 12, 6088 (2004)

18. Hildebrandt, M., Frede, M., Kwee, P., Willke, B., Kracht, D.: Single-frequency master-oscillator photonic crystal fiber amplifier with $148 \mathrm{~W}$ output power. Opt. Express 14, 11071 (2006)

19. Green, M., Keevers, M.: Optical properties of intrinsic Silicon @ 300K. Prog. Photovoltaic Res. Appl. 3, 189 (1995)

20. http://www.rpmclasers.com/Manlight/CW\%20Erbium\%20Fiber\%20Laser.htm

21. Tovstonog, S.V., Kurimura, S., Suzuki, I., Takeno, K., Moriwaki, S., Ohmae, N., Mio, N., Katagai, T.: Thermal effects in high-power cw second harmonic generation in $\mathrm{Mg}$-doped stoichiometric lithium tantalate. Opt. Express 16, 11294 (2008)

22. Samanta, G.K., Kumar, S.C., Ebrahim-Zadeh, M.: Stable, 9.6 W, continuous-wave, single-frequency, fiber-based green source at $532 \mathrm{~nm}$. Opt. Lett. 34, 1561 (2009)

23. Schnabel, R., Harms, J., Strain, K.A., Danzmann, K.: Squeezed light for the interferometric detection of high-frequency gravitational waves. Class. Quantum Gravit. 21, S1045 (2004)

24. Winkler, W., Danzmann, K., Rdiger, A., Schilling, R.: Heating by optical absorption and the performance of interferometric gravitational-wave detectors. Phys. Rev. A 44, 7022 (1991) 
25. Bass, M. (ed.): The Optical Society of America Handbook of Optics, vol. II. McGraw Hill, New York, 33.53 (1995); http://www.lambdaphoto.co.uk/pdfs/Inrad_datasheet_LNB.pdf; http://www.photox. co.uk/linbo3.htm; K. K. Wong Properties of lithium niobate, (IET, 2002) ISBN 0852967993

26. Dmitriev, V.G., Gurzadyan, G.G., Nikogosoyan, D.N.: Handbook of Nonlinear Optical Crystals, Springer Series in Optical Sciences. Springer, Berlin (1999)

27. http://www.crystaltechnology.com/docs/LNopt.pdf

28. Bryan, D.A., Gerson, R., Tomaschke, H.E.: Increase optical damage resistance in lithium niobate. Appl. Phys. Lett. 44, 847 (1984)

29. Kar, S., Choubey, R.K., Sen, P., Bhagavannarayana, G., Bartwal, K.S.: Studies on codoping behavior of $\mathrm{Nd}: \mathrm{Mg}: \mathrm{LiNbO}_{3}$ crystals. Phys. B 393, 37 (2007) (and references)

30. http://www.newfocus.com

31. Karlsson, H.: Fabrication of periodically poled crystals from the KTP family and their applications in nonlinear optics. Thesis, The Royal Institute of Technology, Stockholm (1999). TRITA-FYS 2197. ISSN: 0280-316X

32. Carvajal, J.J., Segonds, P., Pena, A., Zaccaro, J., Boulanger, B., Diaz, F., Aguilo, M.: Structural and optical properties of $\mathrm{RbTiOPO}_{4}: \mathrm{Nb}$ crystals. J. Phys. Condensed Matter 19, 116214 (2007)

33. See for example the KTP and $\mathrm{MgO}: \mathrm{LiNbO}_{3}$ modulators from http://www.newfocus.com

34. Emanueli, S., Arie, A.: Temperature-dependent dispersion equations for $\mathrm{KTiOPO}_{4}$ and $\mathrm{KTiOAsO}_{4}$. Appl. Opt. 42, 6661 (2003)

35. Bass, M. (ed.): The Optical Society of America Handbook of Optics, vol. II. McGraw Hill, New York (1995)

36. http://www.redoptronics.com/KTP-crystal.html

37. Huang, C.H., Shen, H.Y., Zeng, Z.D., Zhou, Y.P., Zeng, R.R., Yu, G.F., Jiang, A.D., Chen, T.B.: Measurment of the total absorption coefficient of a KTP crystal. Opt. Laser Technol. 22, 345 (1990)

38. http://www.lc-solutions.com/product/ktp.php

39. Oseledchik, Y.S., Pisarevsky, A.I., Prosvirnin, A.L., Starshenko, V.V., Svitanko, N.V.: Nonlinear optical properties of the flux grown $\mathrm{RbTiOPO}_{4}$ crystal. Opt. Mater. 3, 237 (1994)

40. Carvajal, J.J., Sole, R., Gavalda, Jna., Massons, J., Rico, M., Zaldo, C., Aguilo, M., Diaz, F.: Growth and characterisation of $\mathrm{RbTiOPO}_{4}: \mathrm{Nd}$ crystals as host for rare earth ions. J. Alloys Compounds 323-324, 231 (2001)

41. Yutsis, I., Kirshner, B., Arie, A.: Temperature-dependent dispersion relations for $\mathrm{RbTiOPO}_{4}$ and $\mathrm{RbTiOAsO}_{4}$. Appl. Phys. B 79, 77 (2004)

42. http://www.redoptronics.com/RTP-crystal.html

43. Mikami, T., Okamoto, T., Kato, K.: Sellmeier and themo-optic dispersion formulas for RbTiOPO 4 . Opt. Mater. doi:10.1016/j.optmat.2009.03.12 (2009)

44. Wu, W.: Instrumentation of the next generation gravitational wave detector: triple pendulum suspension and electro-optic modulator. Thesis, University of Florida (2007)

45. Angert, Nahum, Raicol Crystals Ltd: Personal communication (2009)

46. http://www.redoptronics.com/KTA-crystal.html

47. See for example: http://www.clasers.com/lasercomponents/pockelcell/BBOPockelCell.htm

48. http://www.coherent.com/downloads/BBO_DS.pdf

49. http://bbo-crystal.optical-components.com/bbocrystal-physicalproperties.htm; http://www.unitedcrystals.com/BBOProp.html

50. Khazanov, E., Andreev, N., Babin, A., Kiselev, A., Palashov, O., Reitze, D.H.: Suppression of selfinduced depolarization of high-power laser radiation in glass-based Faraday isolators. J. Opt. Soc. Am B. 17, 99 (2000)

51. Khazanov, E., Andreev, N., Mal'shakov, A., Palashov, O., Poteomkin, A., Sergeev, A.M., Shaykin, A., Zelenogorsky, V., Ivanov, I., Amin, R., Mueller, G., Tanner, D.B., Reitze, D.H.: Compensation of thermally induced modal distortions in Faraday isolators. IEEE J. Quantum Electron. 40, 1500 (2004)

52. Mueller, G., Amin, R., Guagliardo, D., McFeron, Donavan, Lundock, R., Reitze, D.H., Tanner, D.B.: Method for compensation of thermally-induced modal distortions in the input optics components of gravitational wave interferometers. Class. Quantum Gravit. 19, 1793 (2002)

53. Tokita, S., Kawanaka, J., Fujita, M., Kawashima, T., Izawa, Y.: Sapphire-conductive end-cooling of high power cryogenic Yb:YAG lasers. Appl. Phys. B 80, 635 (2005)

54. Mukhin, I.B., Khazanov, E.A.: Use of thin discs in Faraday isolators for high-average power lasers. Quantum Electron. 34, 973 (2004) 
55. Yasuhara, R. III., Tokita, S., Kawanaka, J., Kawashima, T., Kan, H., Yagi, H., Nozawa, H., Yanagitani, T., Fujimoto, Y., Yoshida, H., Nakatsuka, M.: Cryogenic temperature characteristics of Verdet constant on terbium gallium garnet ceramics. Opt. Express 15, 11255 (2007)

56. Zheleznov, D.S., Voitovich, A.V., Mukhin, I.B., Palashov, O.V., Khazanov, E.A.: Considerable reduction of thermooptical distortions in Faraday isolators cooled to $77 \mathrm{~K}$. Quantum Electron. 36, 383 (2006)

57. Zheleznov, D.S., Khazanov, E.A., Mukhin, I.B., Palashov, O.V., Voytovich, A.V.: Faraday isolators with short magnetooptical elements for average laser power up to $100 \mathrm{~kW}$. IEEE J. Quantum Electron. 43, 451 (2007)

58. Mukhin, I., Voitovich, A., Palashov, O., Khazanov, E.: 2.1 Tesla permanent-magnet Faraday isolator for subkilowatt average power lasers. Opt. Commun. 282, 1969 (2009)

59. http://www.mt-berlin.com/charts/chart_07.htm

60. Fratello, V.J., Wolfe, R.: In: Francombe M.H. (ed.) Handbook of Thin Film Devices Magnetic Thin Film Devices, vol. 4, p. 93. Academic Press, New York (2000)

61. Huang, M., Xu, Z.-C.: Wavelength and temperature characteristics of BiYbIG film/YIG crystal composite structure for magneto-optical applications. Appl. Phys. A 81, 193 (2005)

62. Fratello, V.J.: Personal communication (2009)

63. Wolfe, R., Dillon, J.E., Liebeman, R.A., Fratello, V.J.: Broadband magneto-optic waveguide isolator. Appl. Phys. Lett. 57, 960 (1990)

64. Honda, Y., Ishikawa, T., Hibiya, T.: Advances in magneto-optics. J. Magn. Soc. Jpn. 11(Supplement S1), 361 (1987)

65. Tamaki, T., Kaneda, H., Tsushima, K.: $(\mathrm{GdBi})_{3}(\mathrm{FeAR})_{5} \mathrm{O}_{12}$ based Faraday rotator operating at $1.3 \mu \mathrm{m}$. J. Mag. Soc. Jpn. 10, 137 (1986)

66. Dexter, J.L., Landry, J., Cooper, D.G., Reintjes, J.: Ultraviolet optical isolators utilizing KDP-isomorphs. Opt. Commun. 80, 115 (1990)

67. Pye, L.D., Cherukuri, S.C., Mansfield, J., Loretz, T.: The Faraday effect in some noncrystalline fluorides. J. Noncryst. Solids 56, 99 (1983)

68. Acernese, F., et al.: In-vacuum optical isolation changes by heating in a Faraday isolator. Appl. Opt. 47, 5853 (2008)

69. http://ilog.ligo-la.caltech.edu/ilog/pub/ilog.cgi?group=detector\&date_to_view=04/24/2005\& anchor_to_scroll_to=2005:04:24:23:46:45-rana

70. Sun, K.-X., Byer, R.L.: All-reflective Michelson, Sagnac, and Fabry-Perot interferometers based on grating beam splitters. Opt. Lett. 23, 567 (1998)

71. Friedrich, D., Burmeister, O., Bunkowski, A., Clausnitzer, T., Fahr, S., Kley, E.-B., Tünnermann, A., Danzmann, K., Schnabel, R.: Diffractive beam splitter characterization via a power-recycled interferometer. Opt. Lett. 33, 101 (2008)

72. Bunkowski, A., Burmeister, O., Danzmann, K., Schnabel, R., Clausnitzer, T., Kley, E.-B., Tünnermann, A.: Optical Characterization of ultra-high diffraction efficiency gratings. Appl. Opt. 45, 5795 (2006)

73. Bunkowski, A., Burmeister, O., Danzmann, K., Schnabel, R.: Input-Output relations for a three-port grating coupled Fabry-Perot cavity. Opt. Lett. 30, 1183 (2005)

74. Bunkowski, A., Burmeister, O., Danzmann, K., Schnabel, R., Clausnitzer, T., Kley, E.-B., Tünnermann, A.: Demonstration of three-port grating phase relations. Opt. Lett. 31, 2384 (2006)

75. Bunkowski, A., Burmeister, O., Beyersdorf, P., Danzmann, K., Schnabel, R., Clausnitzer, T., Kley, E.-B., Tünnermann, A.: Low-loss grating for coupling to a high-finesse cavity. Opt. Lett. 29, 2342 (2004)

76. Freise, A., Bunkowksi, A., Schnabel, R.: Phase and alignment noise in grating interferometers. N. J. Phys. 9, 433 (2007)

77. Hallam, J., Chelkowski, S., Freise, A., Hild, S., Barr, B., Strain, K.A., Burmeister, O., Schnabel, R.: Coupling of lateral grating displacement to the output ports of a diffractive Fabry-Perot cavity. J. Opt. A Pure Appl. Opt. 11, 085502 (2009)

78. Harry, G.M., Gretarsson, A.M., Saulson, P.R., Kittelberger, S.E., Penn, S.D., Startin, W.J., Rowan, S., Fejer, M.M., Crooks, D.R.M., Cagnoli, G., Hough, J., Nakagawa, N.: Thermal noise in interferometric gravitational wave detectors due to dielectric optical coatings. Class. Quantum Gravit. 19, 897 (2002)

79. Agresti, J., Castaldi, G., DeSalvo, R., Pierro, V., Pinto, I.M.: Optimized multilayer dielectric mirror coatings for gravitational wave interferometers. In: Proc. SPIE, vol. 6286 (2006) 
80. Harry, G.M., Armandula, H., Black, E., Crooks, D.R.M., Cagnoli, G., Hough, J., Murray, P., Reid, S., Rowan, S., Sneddon, P., Fejer, M.M., Route, R., Penn, S.D.: Thermal noise from optical coatings in gravitational wave detectors. Appl. Opt. 45, 1569 (2006)

81. Braginsky, V.B., Vyatchanin, S.P.: Corner reflectors and quantum-non-demolition measurements in gravitational wave antennae. Phys. Lett. A 324, 345 (2004)

82. Gossler, S., Cumpston, J., McKenzie, K., Mow-Lowry, C.M., Gray, M.B., McClelland, D.E.: Coating-free mirrors for high precision interferometric experiments. Phys. Rev. A 76, 053810 (2007)

83. Bunkowski, A., Burmeister, O., Friedrich, D., Danzmann, K., Schnabel, R.: High reflectivity grating waveguide coatings for $1064 \mathrm{~nm}$, Class. Quantum Gravit. 23, 7297 (2006)

84. Brückner, F., Clausnitzer, T., Burmeister, O., Friedrich, D., Kley, E.-B., Danzmann, K., Tünnermann, A., Schnabel, R.: Monolithic dielectric surfaces as new low-loss light-matter interfaces. Opt. Lett. 33, 264 (2008)

85. Sharon, A., Rosenblatt, D., Friesem, A.A.: Resonant grating-waveguide structures for visible and near-infrared radiation. J. Opt. Soc. Am. A 14, 2985 (1997)

86. Moharam, M.G., Gaylord, T.K.: Diffraction analysis of dielectric surface-relief gratings. J. Opt. Soc. Am. 72, 1385 (1982)

87. Brückner, F., Friedrich, D., Clausnitzer, T., Burmeister, O., Britzger, M., Kley, E.-B., Danzmann, K., Tünnermann, A., Schnabel, R.: Demonstration of a cavity coupler based on a resonant waveguide grating. Opt. Express 17, 163 (2009)

88. Chelkowski, S., Hild, A., Freise, A.: Prospects of higher-order Laguerre-Gauss modes in future gravitational wave detectors. Phys. Rev. D 79, 122002 (2009)

89. Gerry, C.C., Knight, P.L.: Introductory Quantum Optics. Cambridge Univ. Press, Cambridge (2004)

90. Jaekel, M.T., Reynaud, S.: Europhys. Lett. 13, 301 (1990)

91. Braginsky, V.B., Khalili, F.Y.: In: Thorne, K.S. Quantum Measurement, Cambridge University Press, Cambridge (1992)

92. Kimble, H.J., Levin, Y., Matsko, A.B., Thorne, K.S., Vyatchanin, S.P.: Phys. Rev. D 65, 022002 (2001)

93. Harms, J., Chen, Y., Chelkowski, S., Franzen, A., Vahlbruch, H., Danzmann, K., Schnabel, R.: Phys. Rev. D 68, 042001 (2003)

94. Slusher, R.E., Hollberg, L.W., Yurke, B., Mertz, J.C., Valley, J.F.: Phys. Rev. Lett. 55, 2409 (1985)

95. Lam, P.K., Ralph, T.C., Buchler, B.C., McClelland, D.E., Bachor, H.A., Gao, J.: Optimisation and transfer of vacuum squeezing from a below threshold optical parametric oscillator. J. Opt. B Quant. Semi. Class. Opt. 1, 469 (1999)

96. McKenzie, K., Grosse, N., Bowen, W.P., Whitcomb, S.E., Gray, M.B., McClelland, D.E., Lam, P.K.: Phys. Rev. Lett. 93, 161105 (2004)

97. Chelkowski, S., Vahlbruch, H., Danzmann, K., Schnabel, R.: Coherent control of broadband vacuum squeezing. Phys. Rev. A 75, 043814 (2007)

98. Vahlbruch, H., Chelkowski, S., Hage, B., Franzen, A., Danzmann, K., Schnabel, R.: Coherent control of vacuum squeezing in the gravitational-wave detection band. Phys. Rev. Lett. 97, 011101 (2006)

99. Vahlbruch, H., et al.: Observation of squeezed light with $10 \mathrm{~dB}$ quantum-noise reduction. Phys. Rev. Lett. 100, 33602 (2008)

100. Goda, K., Miyakawa, O., Mikhailov, E.E., Saraf, S., Adhikari, R., McKenzie, K., Ward, R., Vass, S., Weinstein, A.J., Mavalvala, N.: A quantum-enhanced prototype gravitational-wave detector. Nat. Phys. 4, 472 (2008)

101. Vahlbruch, H., Chelkowski, S., Danzmann, K., Schnabel, R.: Quantum engineering of squeezed states for quantum communication and metrology. N. J. Phys. 9, 371 (2007)

102. McKenzie, K., Mikhailov, E., Goda, K., Lam, P.K., Grosse, N., Gray, M.B., McClelland, D.E.: Quantum noise locking. J. Opt. B Quantum Semiclass. Opt. 7, S421 (2005)

103. McKenzie, K., Shaddock, D.A., McClelland, D.E., Buchler, B.C., Lam, P.K.: Phys. Rev. Lett. 88, 231102 (2002)

104. Vahlbruch, H., Chelkowski, S., Hage, B., Franzen, A., Danzmann, K., Schnabel, R.: Phys. Rev. Lett. 95, 211102 (2005)

105. Schnabel R.: Gravitational wave detectors: squeezing up the sensitivity. Nat. Phys. 4, 440 (2008) (News and Views) 